during the antibiotic treatment of various spirochete infections. It begins 2 to 8 hours after the treatment, as a flu like reaction with high fever and is sometimes associated to an aggravation of the syphilis symptoms. It usually resolves in 24 hours without treatment, but the patients usually receive antipyretic treatment. This reaction appears in 50\% of primary syphilis, and up to $75 \%$ of secondary syphilis. It's very rare in latent syphilis, but can appear in $30 \%$ of neuro-syphilis. There has been only one description of a recurrent JHR, in a patient with a late latent syphilis that had 2 JHR after 2 consecutive penicillin injections. To our knowledge it has not been described in any other syphilis patient.

\section{P2.53 BUSCHKE-LÖWENSTEIN TUMOUR IN ASSOCIATION WITH HPV TYPES 6 AND 11}

D Natasatsekova; Nikolova, M Balabanova, L Miteva, G Pehlivanov. Medical University, Department of Dermatovenereology, Sofia - Bulgaria

\subsection{6/sextrans-2017-053264.229}

Introduction Buschke-Löwenstein tumour (BLT) is a very rare sexually-transmitted disease associated with human papillomavirus (HPV) type 6 and 11, but rare cases of oncogenic HPV types including HPV 16 and HPV 18 were also reported. BLT is located in the genital, anorectal and perianal regions. It is regarded as a type of verrucous carcinoma occurring on anogenital mucosal surfaces where it is locally invasive but displays a benign cytology. Buschke-Löwenstein tumour can be associated with a high rate of recurrence and a risk of malignant transformation to invasive SCC, especially in patients with oncogenic types of HPV.

Methods We report the case of a 59-year-old female patient who addressed our clinic for a large, exophytic, cauliflowerlike tumour involving the vulva, perineum and perianal regions with 20 years duration. The first lesions had been appeared on vulva and after 3 years period they grown slowly and covered perineum and perianal area. They had cauliflower like surface, with different sizes. In some points erosions and yellowish secretion with odour are observed.

Results Histologic examination of a biopsy specimen of large tumour presented nets of well-differentiated squamous cell carcinom, as well as a marked mononuclear cell infiltrate and conspicuous koilocytosis. HPV DNA for 6, 11 types was detected with PCR.

Conclusion The patient was sent to the gynaecology surgery department for excision and remains under the supervision of the dermatology and oncology department for rapid treatment of relapses and early detection of malignant transformation.

\section{P2.54 PENILE VERRUCOUS SQUAMOUS CELL CARCINOMA IN PATIENT WITH NON HODGKIN LYMPHOMA}

M Natasatsekova; Balabanova, L Miteva, G Pehlivanov. Medical University, Department of Dermatovenereology, Sofia - Bulgaria

\subsection{6/sextrans-2017-053264.230}

Introduction Verrucous squamous cell carcinoma (SCC), which was first described in 1948 by Ackermann, was reported in the oral cavity, anus, penis and female genitalia. This carcinoma is a low-grade SCC tumour and exhibits slow invasive growth. Regional lymph node metastases are rare and distant metastases have not been reported yet. Penile verrucous SCC carcinoma represents $5 \%$ to $16 \%$ of all penile SCC and in $33 \%$ of cases is associated with HPV type 6,11. Lack of circumcision, poor hygiene, phimosis, tight prepuce and chronic infection are other important causative factors for penile verrucous SCC carcinoma. We are reporting a case of a 70 yearold male patient who has come to our clinic with enlarging erythematous, exophytic papillary mass with foul smell located on glans penis for four- month duration. The patient is a chain-smoker and immunosuppresed due to the treatment of a non- Hodgkin lymphoma. He reported occurrence of multiple condylomata acuminata on genital area with a long lapse, which was treated with local destructive therapy and electrocoagulation. His medical history includes also ischaemic heart disease and coronary insuffiency.

Methods: Histological examination established verrucous SCC carcinoma - hyperkeratosis, parakeratosis, acanthosis with bulbous downward projections into the dermis and well-differentiated tumour cells with invasion in reticular derma with depth of $2.122 \mathrm{~mm}$ and desmoplastic stromal reaction. Polymerase Chain Reaction for HPV DNA detected HPV type 6.

Results and conclusion The surgical excision and amputation penis partialis in Urology surgical department showed that there was not invasion of the tumour in corpora cavernosa and corpus spongiosum and it was classificated as $\mathrm{T} 1 \mathrm{NxMx}$. The patient remains under the supervision of the dermatology and oncology specialists for eventual relapses.

\section{P2.55 BOWENOID PAPULOSIS}

M Natasatsekova; Balabanova, L Miteva, G Pehlivanov. Medical University, Department of Dermatovenereology, Sofia - Bulgaria

\subsection{6/sextrans-2017-053264.231}

Introduction Bowenoid papulosis (BP) is virally induced disease caused by high risk HPV viruses, the most common type 16 and rarely type $18,32,39,42,48,53,58$. Smoking, early sexual initiation, promiscuity, risk sexualbehavior, uncircumcised sexual partners, immunosuppression, pregnancy, oral contraceptives are other causative factors for BP. The disease affects both sexes equally and is typical of young, sexually active people, aged between 20 and 40.Clinical features of BP are solitary or multiple confluent rapidly increasing papules with red-brown colour and diameter $2-10 \mathrm{~mm}$, with uneven papillary or flat-to verrucous surface. They are localised on external genitalia bilaterally and symmetrically. In men cover foreskin, glans penis, in women labia majora, perianal area. This histology make difficult differential diagnosis with Morbus Bowen in anogenital area. Conducted destructive treatment in outpatient settings is with unsatisfactory therapeutic effect.

Methods We report the case of 45 year old female who addressed our clinic for multiple confluent papules with redbrown colour and diameter 2-10 $\mathrm{mm}$., with uneven papillary or flat-to verrucous surface on external genitalia area bilaterally and symmetrically.

Results Histologic examination of a biopsy specimen established acanthosis, parakerathosis, hyperkerathosis, koilocytosis and atypical cells with hyperchromic bi, multinuclei occupying almost half the thickness of the epidermis to the extent of bowenoid dysplasia. 